\title{
Histological Effect of Basic Fibroblast Growth Factor on Chronic Vocal Fold Scarring in a Rat Model
}

\author{
Ichiro Tateya ${ }^{1} \cdot$ Tomoko Tateya $^{2,3} \cdot$ Jin-Ho Sohn ${ }^{4}$ Diane M. Bless ${ }^{5}$ \\ ${ }^{1}$ Department of Otolaryngology-Head and Neck Surgery, Graduate School of Medicine, ${ }^{2}$ Institute for Virus Research, ${ }^{3}$ The Hakubi Center, Kyoto \\ University, Kyoto, Japan; ${ }^{4}$ Departments of Otorhinolaryngology-Head and Neck Surgery, Kyungpook National University School of Medicine, \\ Daegu, Korea; ${ }^{5}$ Division of Otolaryngology-Head and Neck Surgery, University of Wisconsin-Madison, Madison, WI, USA
}

Objectives. Vocal fold scarring is one of the most challenging laryngeal disorders to treat and there are currently no consistently effective treatments available. Our previous studies have shown the therapeutic potential of basic fibroblast growth factor (bFGF) for vocal fold scarring. However, the histological effects of bFGF on scarred vocal fold have not been elucidated. The aim of this study was to examine the histological effects of bFGF on chronic vocal fold scarring.

Methods. Sprague-Dawley rats were divided into phosphate buffered saline (sham) and bFGF groups. Unilateral vocal fold stripping was performed and the drug was injected into the scarred vocal fold for each group 2 months postoperatively. Injections were performed weekly for 4 weeks. Two months after the last injection, larynges were harvested and histologically analyzed.

Results. A significant increase of hyaluronic acid was observed in the vocal fold of the bFGF group compared with that of the sham group. However, there was no remarkable change in collagen expression nor in vocal fold contraction.

Conclusion. Significant increase of hyaluronic acid by local bFGF injection was thought to contribute to the therapeutic effects on chronic vocal fold scarring.

Keywords. Vocal Cords; Basic Fibroblast Growth Factor 2; Hyaluronic Acid; Collagen

\section{INTRODUCTION}

The extracellular matrix (ECM) of the vocal fold lamina propria (LP) consists of a number of important molecules that contribute to tissue characteristics during oscillation $[1,2]$. The ECM consists of fibrous proteins and interstitial constituents. Collagen and elastin are 2 of the fibrous proteins found in the vocal fold LP and contribute to tissue strength and flexibility. Hyaluronic acid (HA) is a member of the glycosaminoglycan family and is

\footnotetext{
- Received November 19, 2014

Revised December 23, 2014

Accepted January 14, 2015

- Corresponding author: Ichiro Tateya

Department of Otolaryngology-Head and Neck Surgery, Graduate School of Medicine, Kyoto University, 54 Shogoin-Kawahara, Sakyo-ku, Kyoto 606-8507, Japan

Tel: +81-75-751-3346, Fax: +81-75-751-7225

E-mail: tateya@ent.kuhp.kyoto-u.ac.jp
}

abundant in the vocal fold LP. Several studies have shown that HA contributes to the viscoelastic properties of the vocal fold cover and influences tissue viscosity, playing an important role in proper vocal fold vibration $[1,3]$.

Vocal fold scarring is the single greatest cause of poor voice following phonosurgery $[4,5]$. Pathophysiological studies of vocal fold scarring using animal models showed that scarred vocal folds are characterized by increased/disorganized collagen [6-8] and decreased HA $[7,8]$, which destroys the viscoelastic property of the vocal fold $[2,6]$. The altered structure changes the biomechanical properties of the vocal fold often resulting in a severe dysphonia that is difficult to treat surgically or by behavioral modification. Many injectable materials such as bovine [9], autologous [10], homologous collagen [11], or autologous fat [12] have been used for the treatment of vocal fold scarring. However, the therapeutic effects of these materials on vocal fold scarring still have been limited.

Copyright (C) 2016 by Korean Society of Otorhinolaryngology-Head and Neck Surgery.

This is an open-access article distributed under the terms of the Creative Commons Attribution Non-Commercial License (http://creativecommons.org/licenses/by-nc/4.0)

which permits unrestricted non-commercial use, distribution, and reproduction in any medium, provided the original work is properly cited. 
Vocal fold LP consists of ECM components and cells consisting mostly of fibroblasts, which produce ECM components. It is reasonable to assume that an appropriate control of fibroblasts in terms of ECM production may be necessary to digest the excessive collagen deposits, synthesize HA, and restore the scarred vocal fold to normal [13]. Growth factor is a potent regulatory element that affects fibroblasts and their functions. Basic fibroblast growth factor (bFGF) stimulates cell growth of fibroblasts and modulates ECM synthesis $[14,15]$. In vitro study using rat vocal fold fibroblasts suggested that bFGF stimulates HA production and suppresses the production of collagen [16] and in vivo study using a rat model of chronic vocal fold scarring showed that local injection of bFGF improved the phonatory function of scarred vocal folds [17]. Local bFGF injection has been applied on vocal fold scar and sulcus in human cases and reported to improve some of phonatory parameters [18]. Though there have been those reports that suggested the therapeutic effect of bFGF on vocal fold scarring, histological effects of chronic vocal fold scarring treated by bFGF have not been documented. The purpose of this study was to examine the therapeutic effects of bFGF on chronic vocal fold scarring using a rat model.

Tateya et al. [7], clarified the characteristics of rat vocal fold scarring by examining the alteration of key components in the ECM and showed that the tissue remodeling process in scarred vocal folds slows down around 2 months after wounding. It suggested that chronic vocal fold scarring can be obtained 2 months after vocal fold injury and therefore the scarring in vocal folds 2 months after wounding was defined as 'chronic' vocal fold scarring.

\section{MATERIALS AND METHODS}

\section{Rat videolaryngoscopic surgery}

Eleven Sprague-Dawley male rats (400-450 g in weight) were involved in the study. Rat vocal fold stripping was performed as previously described $[7,8]$. In short, rats were anesthetized with an intraperitoneal injection of ketamine $(90 \mathrm{mg} / \mathrm{kg}$; Animal Health, Fort Dodge, IA, USA) and xylazine $(9 \mathrm{mg} / \mathrm{kg}$; Phoenix, St. Joseph, MO, USA). Atropine sulfate $(0.05 \mathrm{mg} / \mathrm{kg}$; Phoenix) was injected intraperitoneally to reduce the secretion of saliva and sputum in the laryngeal lumen. The animals were placed on an operating platform in a near-vertical position $[19,20]$. A suspension microlaryngoscope, fabricated from 1-mm diameter steel wire, was inserted though the mouth to maintain the surgical field. Vocal folds were visualized using a 1.9-mm diameter telescope with an angle of 25 degrees (Richard Wolf, Vernon Hills, IL, USA). Using a 25-G spinal needle (Tyco Healthcare, Mansfield, MA, USA) and microforceps, unilateral vocal fold stripping was performed until the thyroarytenoid muscle was exposed. The other side was kept intact and used as a control. All rats recovered from the anesthesia.

\section{bFGF injection into scarred vocal folds}

Two months postoperatively, rats were randomly divided into sham group and bFGF group. Six rats were sham and 5 rats were bFGF group. Ten microliters of phosphate buffered saline (PBS) or bFGF (100 ng in $10 \mu \mathrm{L}$ of PBS, Sigma-Aldrich, St. Louis, MO, USA) were injected into the scarred vocal folds of each group, respectively. Injections were performed weekly for 4 weeks using 27G Hamilton's syringe (Hamilton, Reno, NV, USA) under the videolaryngoscope. We chose this procedure in order to share the experimental protocol with our previous study [17], though less frequent injection would be more practical for considering clinical application to human body.

\section{Tissue preparation and histological confirmation of the injury}

Two months after the last injection, the rats were humanely euthanized.The rats were anesthetized with a mixture of ketamine $(90 \mathrm{mg} / \mathrm{kg})$ and xylazine $(9 \mathrm{mg} / \mathrm{kg})$ and Euthasol $(0.22 \mathrm{~mL} / \mathrm{kg}$; Diamond Animal Health Inc., Des Moines, IA, USA) was injected intracardially. Whole larynges were harvested, soaked in embedding medium (O.C.T. compound, Tissue-Tek, Kyoto, Japan), frozen quickly with a combination of acetone and dry ice, and kept in a deep freezer $\left(-80^{\circ} \mathrm{C}\right)$. Ten-micron thick cryostat coronal sections of the vocal folds were prepared, air-dried, and stored at $-20^{\circ} \mathrm{C}$ until use. Slices were numbered from the posterior end to the anterior end of the membranous portion of vocal folds. The slides, including the middle one third of the vocal folds, were used for histological analysis.

\section{Staining method}

Masson's Trichrome staining (American Master Tech ScientificInc., Lodi, CA, USA) was used to detect collagen. Alcian Blue staining (Newcomer Supply Inc., Middleton, WI, USA) and a hyaluronidase digestion technique were used to detect $\mathrm{HA}$, and HA was identified by comparing the sections without digestion to those with digestion. For the hyaluronidase digestion procedure, $50 \mathrm{mg}$ of bovine testicular hyaluronidase (Sigma-Aldrich) was diluted in 100-mL PBS. Each section was incubated in this solution for 1 hour at $37^{\circ} \mathrm{C}$. Next, the sections were stained with Alcian Blue (pH 2.5). HA was identified by comparing the sections with and without digestion.

The stained area of each scarred and control vocal fold in each slice was analyzed using an image analysis system specifically developed for the quantification of histological images $[7,8,13,21]$. The LP of each vocal fold was examined at original magnifications $\times 20$. Images were captured with a Nikon Eclipse E600 microscope (Nikon, Melville, NY, USA) and a Pixcera color camera (model PVC C, Los Gatos, CA, USA). Metamorph Image Analysis Software (Universal Imaging, West Chester, PA, USA) measured the density of the stained regions from the LP. The ratio (\%) of pixels in the stained area relative to the total number of pixels in the LP was provided as an indicator of each molecule's density and regarded as the density of HA or colla- 
gen. To minimize measurement bias, all measurements were carried out in a blinded fashion such that the examiner who analyzed the images had no information of the groups to which the specimens belonged.

Two slices for each stain from each animal were prepared and measured, and the results were averaged for each vocal fold. Ratios of HA and collagen in the scarred vocal folds compared to the ratios in the contralateral control vocal folds were calculated, with the average stained ratio of collagen and HA for each animal.

Contraction of the scarred vocal folds was measured as previously described [21]. In brief, the thickness of the LP was determined by measuring the distance from the free edge of the vocal fold down to the muscle layer at $10 \times$ microscopic power using Adobe Photoshop Image Analysis Software 7.0 (Adobe Systems Inc., San Jose, CA, USA). The measurements were performed on both normal and treated sides, and the thickness of the treated LP was represented by the ratio of pixel size measured on the treated side per that on the normal side. To minimize measurement bias, all measurements were carried out in a blinded fashion. Statistical comparisons were performed between sham and growth factor-treated groups using an unpaired $t$-test. A $P$-value $<0.05$ was considered statistically significant.

\section{Statistical analysis}

The specimens of sham, bFGF, and HGF groups were used for statistical analysis. Mann-Whiney $U$-test was used to calculate differences between treated and normal sides within each group, and between sham and bFGF groups. Differences at $P<0.05$ were regarded as statistically significant. Interjudge reliability was assessed using Spearman correlation. Five percent of these speci- mens were selected at random and remeasured. Initial measures were significantly correlated with repeated measures (image analysis; $r=0.83$ ).

\section{Ethics}

This study was performed in accordance with the PHS Policy on Humane Care and Use of Laboratory Animals, the National Institutes of Health Guide for the Care and use of Laboratory Animals, and the Animal Welfare Act (7 U.S.C. et seq.); the animal use protocol was approved by the Institutional Animal Care and use Committee of University of Wisconsin-Madison.

\section{RESULTS}

\section{HA expression}

Dense blue stain was found chiefly in the intermediate and deep layer of the LP of the normal vocal folds (Fig. 1). The sham control treated with PBS injection showed less blue stain in the intermediate and deep layer whereas bFGF-treated vocal folds revealed a dense blue stained area in the intermediate and deep layer of the LP (Fig. 1). Because this blue stain in the LP was not observed in the specimens treated with bovine testicular hyaluronidase, it was thought to be HA.

Statistical analysis showed less HA $(P<0.01)$ in sham compared to the normal vocal folds whereas the bFGF group showed no significant difference (Fig. 2). Vocal folds treated with bFGF had significantly higher HA $(P<0.01)$ than sham-treated vocal folds.
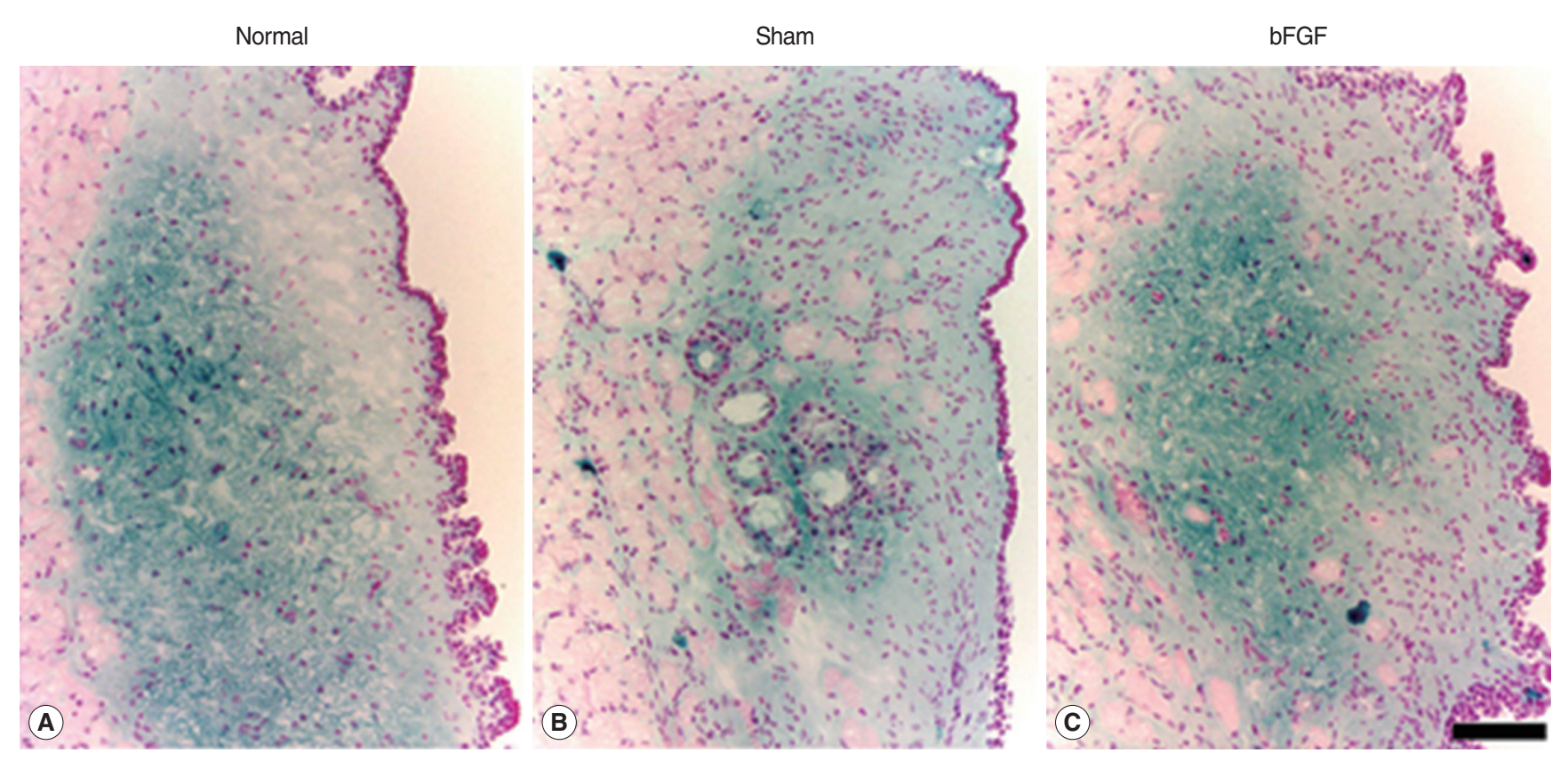

Fig. 1. Representative samples of hyaluronic acid expression (blue) in normal (A) and treated groups (B, C) stained by Alcian Blue stain. The scale bar at the bottom of the figure indicates $50 \mu \mathrm{m}$. bFGF, basic fibroblast growth factor. 


\section{Collagen expression}

Collagen was found mainly in the deep layer of the LP of the normal vocal folds (Fig. 3). In the sham vocal folds, denser collagen was found in all layers of the scarred LP and the collagen deposition was similar in bFGF-treated vocal folds (Fig. 3). There was a significant difference $(P<0.01)$ between normal and treated groups and no significant difference between sham and bFGF groups (Fig. 4).

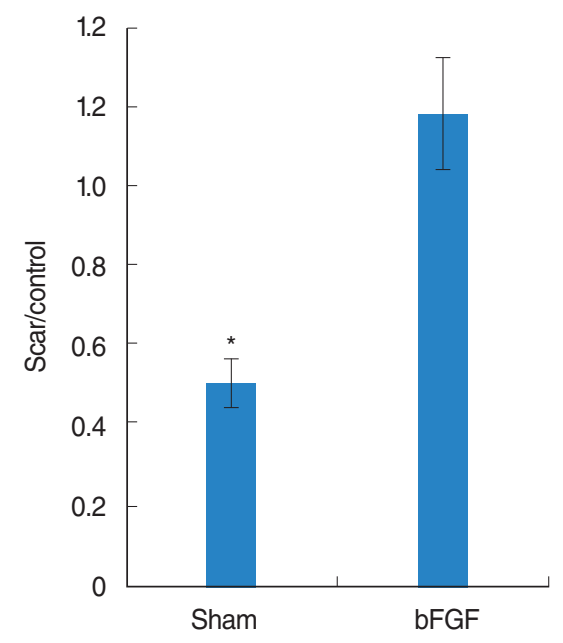

Fig. 2. The average ratio of hyaluronic acid (HA) seen with Alcian Blue staining in the treated vocal fold compared to the ratio in the contra lateral normal vocal fold. Sham groups showed a significant lower HA level compared to the normal control whereas basic fibroblast growth factor (bFGF) group showed no significant difference. *Significant difference $(P<0.01)$ from normal control.

\section{LP thickness}

The thickness of the LP in every treated group was significantly thinner $(P<0.01)$ than that of the normal vocal folds, and there was no significant difference between sham and bFGF groups (Fig. 5).

\section{DISCUSSION}

In vitro study using fibroblasts from skin [22], gingiva [14], periodont [15], and vocal folds [16] revealed that bFGF stimulates

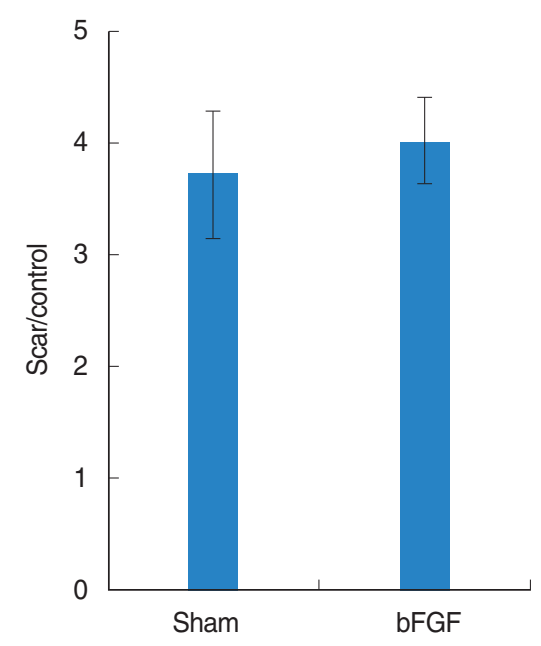

Fig. 4. The average ratio of collagen seen with Trichrome staining in the treated vocal fold compared to the ratio in the contra lateral normal vocal fold. bFGF, basic fibroblast growth factor.
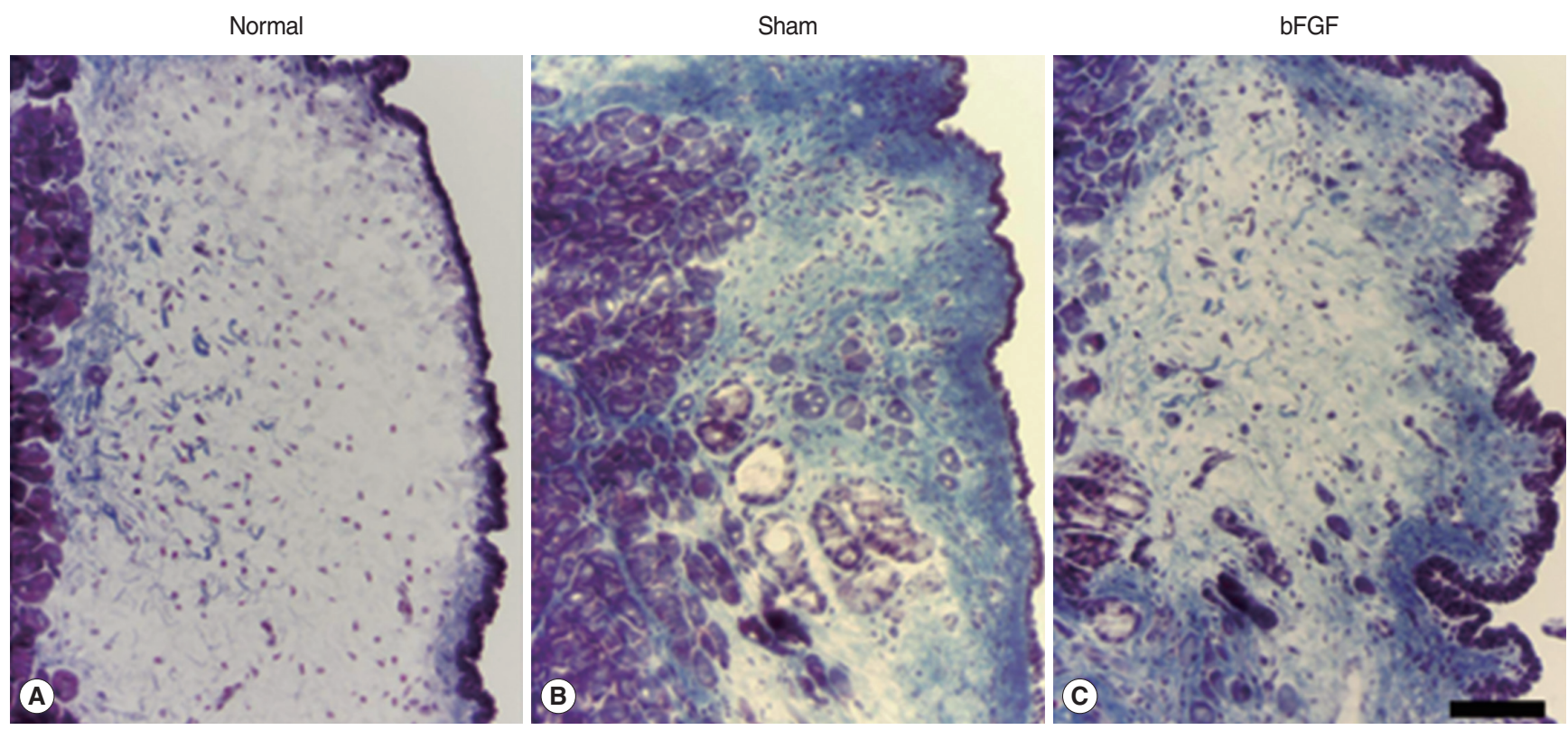

Fig. 3. Representative samples of collagen expression (blue) in normal (A) and treated groups (B, C) stained by Trichrome stain. The scale bar at the bottom of the figure indicates $50 \mu \mathrm{m}$. bFGF, basic fibroblast growth factor. 


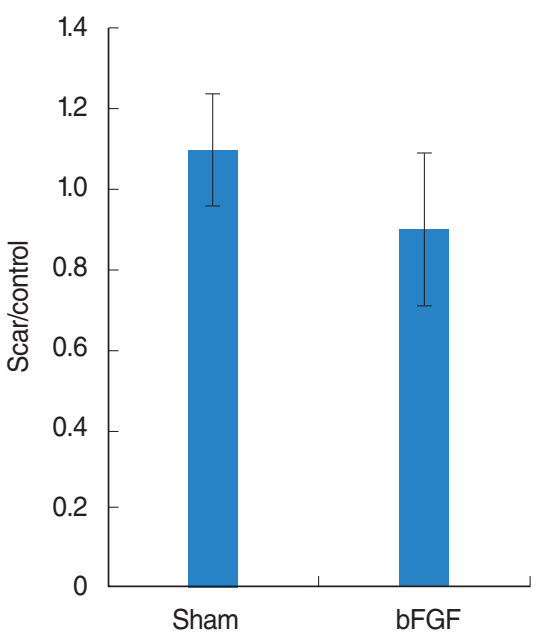

Fig. 5. Thickness of lamina propria in treated groups compared to the contralateral normal side. bFGF, basic fibroblast growth factor.

HA production and decreases collagen type I production. The present in vivo study demonstrated the significant effects on HA increase and no remarkable effect on reduction of collagen and contraction in bFGF treated chronic vocal fold scarring. Similar results have been observed in a rat model of aged vocal folds and bFGF injection into aged vocal folds significantly increased the HA but showed no effect on collagen [23].

We previously demonstrated that local injection of bFGF improved the phonatory function of rat vocal folds suffering chronic scar [17]. Moreover, local bFGF injection into human vocal fold scar and sulcus improved some of the phonatory parameters, and the dissection of scar tissue and the implant of gelatin sponge with bFGF led to better phonatory function than local bFGF injection [18]. Considering these previous studies, HA increase in scarred vocal folds by local bFGF injection was thought to cause therapeutic effect on phonatory function to some extent. It is also suggested that regenerative surgical procedures such as the dissection of scar tissue and the implant of gelatin sponge with bFGF are necessary to restore better phonatory function because local bFGF injection cannot lessen collagen deposition and contraction.

Despite the significant effect on HA, bFGF showed no remarkable effect on reduction of collagen and contraction on chronic vocal fold scarring, despites of the previous in vitro study; bFGF downregulated procollagen I expression in fibroblasts from rat vocal folds [16]. Fibroblasts from scarrd vocal folds may have different properties of producing ECM from those from normal vocal folds. Our previous study showed that collagen types I and III are distributed in the vocal fold LP and that collagen type III is dominant in chronic vocal fold scarring, probably due to the dominance of collagen type III of normal vocal fold LP [7]. The dominance of collagen type III in the chronic vocal fold scarring might result in the insufficient collagen reduction.

In conclusion, the present study confirmed that local bFGF injection led the desirable histological change, HA increase, by using a rat model of chronic vocal fold scarring. It was thought to contribute to the therapeutic effects on chronic vocal fold scarring.

\section{CONFLICT OF INTEREST}

No potential conflict of interest relevant to this article was reported.

\section{ACKNOWLEDGMENTS}

This study was supported by the grant NIDCD RO1DC4428 from the National Institutes of Health, and a grant from the Ministry of Health, Labour, and Welfare (Japan), and a grant from the Ministry of Education, Culture, Sports, Science, and Technology (Japan). The authors thank Dr. Xinhong Lim, for his help for the image analysis, and Ms. Jessica Tiede, Mrs. Maia N. Braden, and Ms. Bridget Ruth Welbourne for their assistance in preparation of our manuscript.

\section{REFERENCES}

1. Gray SD, Titze IR, Alipour F, Hammond TH. Biomechanical and histologic observations of vocal fold fibrous proteins. Ann Otol Rhinol Laryngol. 2000 Jan;109(1):77-85.

2. Thibeault SL, Gray SD, Bless DM, Chan RW, Ford CN. Histologic and rheologic characterization of vocal fold scarring. J Voice. 2002 Mar;16(1):96-104.

3. Chan RW, Gray SD, Titze IR. The importance of hyaluronic acid in vocal fold biomechanics. Otolaryngol Head Neck Surg. 2001 Jun; 124(6):607-14.

4. Benninger MS, Alessi D, Archer S, Bastian R, Ford C, Koufman J, et al. Vocal fold scarring: current concepts and management. Otolaryngol Head Neck Surg. 1996 Nov;115(5):474-82.

5. Woo P, Casper J, Colton R, Brewer D. Diagnosis and treatment of persistent dysphonia after laryngeal surgery: a retrospective analysis of 62 patients. Laryngoscope. 1994 Sep;104(9):1084-91.

6. Rousseau B, Hirano S, Scheidt TD, Welham NV, Thibeault SL, Chan RW, et al. Characterization of vocal fold scarring in a canine model. Laryngoscope. 2003 Apr;113(4):620-7.

7. Tateya T,Tateya I, Sohn JH, Bless DM. Histologic characterization of rat vocal fold scarring. Ann Otol Rhinol Laryngol. 2005 Mar;114(3): 183-91.

8. Tateya T, Tateya I, Sohn JH, Bless DM. Histological study of acute vocal fold injury in a rat model. Ann Otol Rhinol Laryngol. 2006 Apr;115(4):285-92.

9. Ford CN, Bless DM, Campbell D. Studies of injectable soluble collagen for vocal fold augmentation. Rev Laryngol Otol Rhinol (Bord). 1987;108(1):33-6.

10. Ford CN, Staskowski PA, Bless DM. Autologous collagen vocal fold injection: a preliminary clinical study. Laryngoscope. 1995 Sep; 105(9 Pt 1):944-8.

11. Kriesel KJ,Thiebault SL, Chan RW, Suzuki T,VanGroll PJ, Bless DM, 
et al. Treatment of vocal fold scarring: rheological and histological measures of homologous collagen matrix. Ann Otol Rhinol Laryngol. 2002 Oct;111(10):884-9.

12. Wexler DB, Jiang J, Gray SD, Titze IR. Phonosurgical studies: fat-graft reconstruction of injured canine vocal cords. Ann Otol Rhinol Laryngol. 1989 Sep;98(9):668-73.

13. Hirano S, Bless DM, Nagai H, Rousseau B, Welham NV, Montequin DW, et al. Growth factor therapy for vocal fold scarring in a canine model.Ann Otol Rhinol Laryngol. 2004 Oct;113(10):777-85.

14. Hong HH, Trackman PC. Cytokine regulation of gingival fibroblast lysyl oxidase, collagen, and elastin. J Periodontol. 2002 Feb;73(2): 145-52.

15. Palmon A, Roos H, Reichenberg E, GrosskopA, Bar Kana I, Pitaru S, et al. Basic fibroblast growth factor suppresses tropoelastin gene expression in cultured human periodontal fibroblasts. J Periodontal Res. 2001Apr;36(2):65-70.

16. Suehiro A, Hirano S, Kishimoto Y, Tateya I, Rousseau B, Ito J. Effects of basic fibroblast growth factor on rat vocal fold fibroblasts. Ann Otol Rhinol Laryngol. 2010 Oct;119(10):690-6.

17. Welham NV, Montequin DW, Tateya I, Tateya T, Choi SH, Bless DM. A rat excised larynx model of vocal fold scar. J Speech Lang Hear
Res. 2009 Aug;52(4):1008-20.

18. Hirano S, Mizuta M, Kaneko M, Tateya I, Kanemaru S, Ito J. Regenerative phonosurgical treatments for vocal fold scar and sulcus with basic fibroblast growth factor. Laryngoscope. 2013 Nov;123(11): 2749-55.

19. Inagi K, Connor NP, Ford CN, Schultz E, Rodriquez AA, Bless DM, et al. Physiologic assessment of botulinum toxin effects in the rat larynx. Laryngoscope. 1998 Jul;108(7):1048-54.

20. SuzukiT, Connor NP, Lee K, Leverson G, Ford CN. Laryngeal-respiratory kinematics are impaired in aged rats. Ann Otol Rhinol Laryngol. 2002 Aug;111(8):684-9.

21. Hirano S, Bless DM, Rousseau B, Welham N, Montequin D, Chan RW, et al. Prevention of vocal fold scarring by topical injection of hepatocyte growth factor in a rabbit model. Laryngoscope. 2004 Mar;114(3):548-56.

22. Heldin P, Laurent TC, Heldin CH. Effect of growth factors on hyaluronan synthesis in cultured human fibroblasts. Biochem J. 1989 Mar;258(3):919-22.

23. Hirano S, Bless DM, del Río AM, Connor NP, Ford CN. Therapeutic potential of growth factors for aging voice. Laryngoscope. 2004 Dec; 114(12):2161-7. 